\title{
DO VAN HOVE SINGULARITIES IN LEADS INFLUENCE TUNNELING CURRENT THROUGH QUANTUM DOT?
}

\author{
M. Krawiec, T. Domańsiki and K.I. WysokińsKi \\ Institute of Physics, Maria Curie-Skłodowska University \\ Radziszewskiego 10 A, 20-031 Lublin, Poland
}

\begin{abstract}
Using the Landauer type formula for the current through quantum dot we have studied the influence of the singularities in the density of states in leads on the differential conductance of the system and answered affirmatively the above question. The presence of Van Hove singularities in the leads may induce an additional structure on the conductance vs. voltage curve. The dot itself has been described by the single impurity Anderson model with a very strong "on-dot" Coulomb repulsion. We also simulate the effect of electron-phonon interaction in the dot by allowing for the fluctuations of the "impurity" energy level.
\end{abstract}

PACS numbers: $73.40 . \mathrm{Gk}, 72.15 . \mathrm{Qm}, 71.27 .+\mathrm{a}$

It has been predicted theoretically in a number of papers [1] that the quantum dot at a sufficiently low temperature should show all aspects of the Kondo physics known from solutions of the single impurity problem [2]. Recently these predictions have been confirmed in a beautiful experiment [3], which used a single electron transistor (SET) fabricated in a two-dimensional electron gas of the GaAs/AlGaAs heterostructure.

The mesoscopic nature of the impurity in SET makes it possible to study new phenomena not accessible in previous experiments even on very diluted systems. In the latter case one always deals with a number of impurities statistically distributed inside the sample. The new possibilities include the nature of the leads: (i) they can be kept at different chemical potentials which allows for a study of nonequilibrium transport [4], (ii) can be metallic, superconducting [5] or contain Tomonaga-Luttinger quasiparticle liquids [6] as realised in edge states of the quantum Hall effect [7], etc. One can change the properties of the dot itself by coupling the electrons on the dot to external time dependent field and study the frequency dependent conductance of the system [8].

The purpose of the present work is to study the influence of the band structure and in particular Van Hove singularities in the leads on the tunneling current 
through quantum dot. Thus we start our discussion with the Anderson Hamiltonian [9]

$$
\begin{aligned}
H= & \sum_{\lambda k \sigma} \varepsilon_{\lambda k} c_{\lambda k \sigma}^{+} c_{\lambda k \sigma}+E_{\mathrm{d}} \sum_{\sigma} d_{\sigma}^{+} d_{\sigma}+U n_{\uparrow} n_{\downarrow} \\
& +\sum_{\lambda k \sigma}\left(V_{\lambda k} c_{\lambda k \sigma}^{+} d_{\sigma}+\text { h.c. }\right) .
\end{aligned}
$$

Here $\lambda=\mathrm{R}, \mathrm{L}$ denote the right (R) or left $(\mathrm{L})$ lead in the system. The parameters have the following meaning: $c_{\lambda k \sigma}^{+}\left(c_{\lambda k \sigma}\right)$ denote creation (annihilation) operator for a conduction electron with wave vector $k$, spin $\sigma$ in the lead $\lambda, V_{\lambda k}$ is the hybridization matrix element between conduction electron of energy $\varepsilon_{\lambda k}$ in the lead $\lambda$ and localised electron on the dot. $E_{\mathrm{d}}$ is the single particle energy at the dot. Here $n_{\uparrow}=d_{\uparrow}^{+} d_{\uparrow}$ is the number operator for localised electrons with spin up and $U$ is the (repulsive) interaction energy between two localised electrons. In the following we shall study the $U=\infty$ limit. In this limit on the dot there can be at most a single electron with the energy $E_{\mathrm{d}}$.

From the experimental point of view it is differential conductance which plays an important role. To calculate it we first need an expression for the current across the dot when the energy difference $\mathrm{eV}=\mu_{L}-\mu_{R}$ is applied to the leads. Here $\mu_{\lambda}$ denotes chemical potential in the lead $\lambda$. We use here the Landauer type formula [10] which reads

$$
J=\frac{e}{\hbar} \sum_{\sigma} \int \mathrm{d} \omega\left[f_{\mathrm{L}}(\omega)-f_{\mathrm{R}}(\omega)\right] \frac{\Gamma_{\sigma}^{\mathrm{L}}(\omega) \Gamma_{\sigma}^{\mathrm{R}}(\omega)}{\Gamma_{\sigma}^{\mathrm{L}}(\omega)+\Gamma_{\sigma}^{\mathrm{R}}(\omega)}\left(\frac{-1}{\pi}\right) \operatorname{Im} G_{\sigma}\left(\omega+\mathrm{i} 0^{+}\right) .
$$

Here $f_{\lambda}(\omega)$ denotes the Fermi distribution function for the lead $\lambda$ with the chemical potential $\mu_{\lambda}, G_{\sigma}(\omega)$ is the impurity Green function and $\Gamma^{\lambda}(\omega)=2 \pi \sum_{k \lambda}\left|V_{\lambda k}\right|^{2}$ $\times \delta\left(\omega-\varepsilon_{\lambda k}\right)$ is the effective coupling of localised electron to conduction band. To study the effect of Van Hove singularities in leads on the current $J$ and conductance $G=\mathrm{d} J / \mathrm{d} V$, we have assumed the conduction electron energies

$$
\varepsilon_{\lambda k}=\varepsilon_{k}=-(D / 4)\left(\cos k_{x} a+\cos k_{y} a\right),
$$

characteristic of two-dimensional tight binding spectrum, which is known to possess the logarithmic singularity in the middle of the band, which the width we denote by $D$ and take as an energy unit in the following.

To calculate the impurity Green function we used Hubbard-I [11] type of decoupling and obtained

$$
G_{\sigma}(\omega)=\frac{\left(1-\left\langle n_{-\sigma}\right\rangle\right)}{\omega-E_{\mathrm{d}}-\Sigma_{\sigma}(\omega)}
$$

with

$$
\Sigma_{\sigma}(\omega)=\sum_{\lambda k}\left|V_{\lambda k}\right|^{2} \frac{1+f\left(\varepsilon_{\lambda k}\right)}{\omega-\varepsilon_{\lambda k}+\mathrm{i} 0} .
$$

To get the density of states on the dot (equal to $(-1 / \pi) \operatorname{Im} G_{\sigma}(\omega+\mathrm{i} 0)$ ) we have solved Eqs. (4) and (5) self-consistently for $V_{\lambda k}=V_{0}=$ const and for three different bar densities of states (DOS) $\rho^{\lambda}(\varepsilon)=(1 / N) \sum_{k} \delta\left(\varepsilon-\varepsilon_{\lambda k}\right)$ in the conducting leads. We have performed the calculations for (a) constant, (b) Lorentzian and (c) "Van 
Hove" DOS. The last one is obtained for the spectrum given in Eq. (3). It is known to possess Van Hove singularity in the middle of the band. Figure 1 shows an effect of the Van Hove singularity in leads on the density of states of the electrons on a dot. Various curves refer to three DOS as indicated. In the case of the band structure (3) the Van Hove singularity of the left lead has been shifted by $0.1 D$ below and right one the same amount above the Fermi level of the system. In the DOS one observes a clear structure at energies corresponding to the positions of singularities.

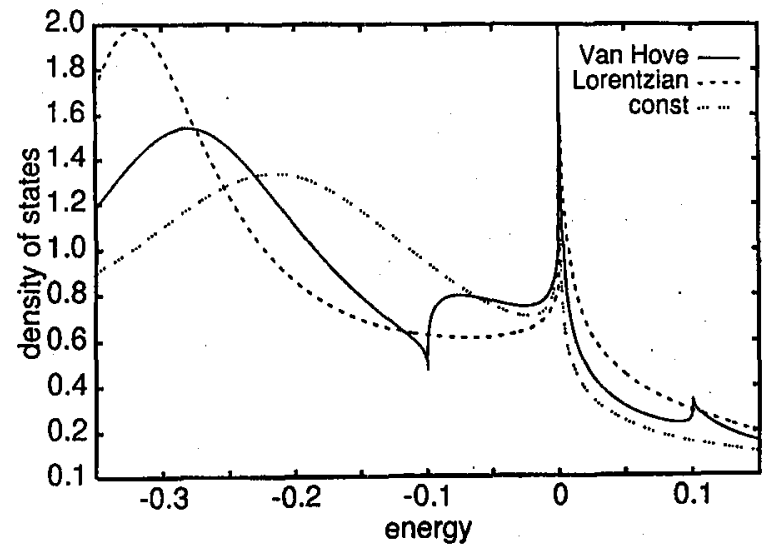

Fig. 1. The density of states on the dot calculated for $V_{0}=0.1 D, T=0.0001 D$ and for three different spectra of electrons in the leads. Note the structure due to Van Hove singularities and the robustness of the coherent (Kondo) feature at the Fermi level (zero of energy).

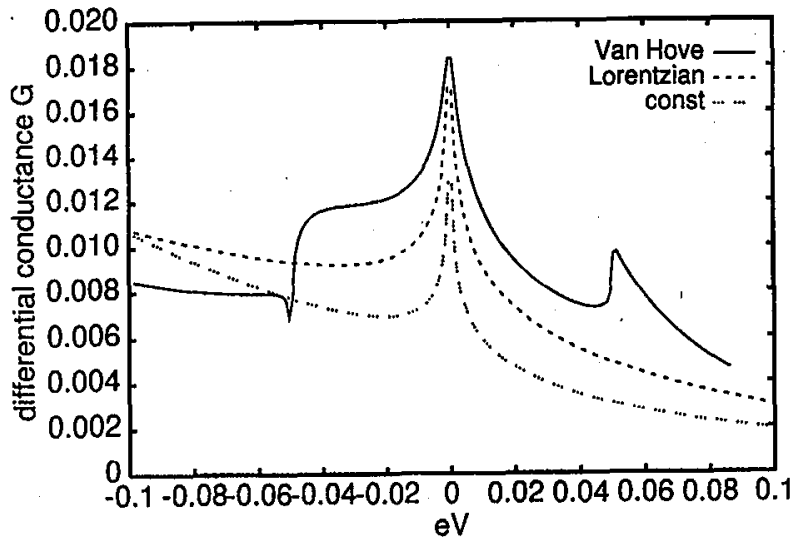

Fig. 2. The differential conductance $G$ (in units of $e^{2} / h$ ) for three spectra of electrons in the leads. The Van Hove singularity affects the conductance and produces additional structures around the Fermi level. 
Figure 2 shows the corresponding differential conductance spectra. One observes marked changes of the tunneling current and the differential conductance due to the presence of the Van Hove singularities (now shifted by $\pm 0.05 \mathrm{D}$ ). The dip seen below Fermi level $\left(\mu_{\mathrm{L}}-\mu_{\mathrm{R}}<0\right)$ is due to the real part of the self-energy, while that above is connected with the imaginary part, which in turn is proportional to the lead DOS.

The electrons in the dot are subject to various scattering processes. In particular there exists electron-ion interaction. The adiabatic approximation allows for solving the electronic eigenproblem for frozen configurations of ions. Each configuration of ions will thus give slightly different electron wave functions and energies $E_{\mathrm{d}}$ which requires the averaging over ionic positions. Here we propose the following approach. The electron-ion interaction modifies the energy level $E_{\mathrm{d}} \rightarrow E_{\mathrm{d}}+\eta$. We assume that $\eta$ has a Gaussian distribution $P(\eta)=(2 \pi \alpha)^{-1 / 2} \exp \left(-\eta^{2} / 2 \alpha\right)$ with (in principle temperature dependent [12]) width $\alpha$ and that the averaging over ionic positions is equivalent to averaging over $\eta$.

The results of the calculations which, because of the lack of space, are not displayed here show the robustness of the coherent feature in the density of states. The other parts of the spectrum are smeared out as expected.

In conclusion we have studied the effect of Van Hove singularity in the leads on the tunneling current across quantum dot. We observed the marked structure due to the singularities which shows up not only in the density of states but also on the nonlinear conductance versus voltage curves. For some parameters this leads to additional structures. The structure, however, displays a completely different temperature dependence. The details of these studies will be presented elsewhere.

This work has been partially supported by the Committee for Scientific Research under the grant 2P03B 03111.

\section{References}

[1] T.-K. Ng, P.A. Lee, Phys. Rev. Lett. 61, 1768 (1988); L.I. Glazman, M.E. Raikh, JETP Lett. 47, 452 (1988).

[2] A.C. Hewson, The Kondo Problem to Heavy Fermions, Cambridge University Press, Cambridge 1993.

[3] D. Goldhaber-Gordon, H. Shtrikman, D. Mahalu, D. Abusch-Magder, U. Meirav, M.A. Kastner, Nature 391, 1569 (1998).

[4] Y. Meir, N.S. Wingreen, P.A. Lee, Phys. Rev. Lett. 70, 2601 (1993).

[5] K. Kang, Phys. Rev. B 57, 11891 (1998); R. Fazio, R. Raimondi, Phys. Rev. Lett. 80, 2913 (1998).

[6] A. Furasaki, Phys. Rev. B 57, 7141 (1998).

[7] K. von Klitzing, Rev. Mod. Phys. 58, 519 (1985).

[8] P. Nordlander, N.S. Wingreen, Y. Meir, D.C. Langreth, preprint cond-mat/9801241.

[9] P.W. Anderson, Phys. Rev. 124, 41 (1961).

[10] Y. Meir, N.S. Wingreen, Phys. Rev. Lett. 68, 2512 (1992).

[11] J. Hubbard, Proc. R. Soc. Lond. A 276, 238 (1963).

[12] K.I. Wysokiński, J. Phys. C 11, 291 (1978). 